Algebraic $8 \mathcal{G}$ Geometric $\mathcal{T}$ opology

Volume 4 (2004) 151-175

Published: 24 March 2004

ATG

\title{
Sur la réalisation des modules instables
}

\author{
DONGHUA JiAng
}

\begin{abstract}
In this article, we give some conditions on the structure of an unstable module, which are satisfied whenever this module is the reduced cohomology of a space or a spectrum. First, we study the structure of the sub-modules of $\Sigma^{s} \tilde{H}^{*}\left(B(\mathbb{Z} / 2)^{\oplus d} ; \mathbb{Z} / 2\right)$, i.e., the unstable modules whose nilpotent filtration has length 1 . Next, we generalise this result to unstable modules whose nilpotent filtration has a finite length, and which verify an additional condition. The result says that under certain hypotheses, the reduced cohomology of a space or a spectrum does not have arbitrary large gaps in its structure. This result is obtained by applying Adams' theorem on the Hopf invariant and the classification of the injective unstable modules.

This work was carried out under the direction of L. Schwartz.

Résumé Dans cet article, on donne des restrictions sur la structure d'un module instable, qui doivent être vérifiées pour que celui-ci soit la cohomologie réduite d'un espace ou d'un spectre. On commence par une étude sur la structure des sous-modules de $\Sigma^{s} \tilde{H}^{*}\left(B(\mathbb{Z} / 2)^{\oplus d} ; \mathbb{Z} / 2\right)$, i.e., les modules instables dont la filtration nilpotente est de longueur 1 . Ensuite, on généralise le résultat aux modules instables dont la filtration nilpotente est de longueur finie, et qui vérifient une condition supplémentaire. Le résultat dit que sous certaines hypothèses, la cohomologie réduite d'un espace ou d'un spectre ne contient pas de lacunes de longueur arbitrairement grande. Ce résultat est obtenu par application du célèbre théorème d'Adams sur l'invariant de Hopf et de la classification des modules instables injectifs.

Ce travail est effectué sous la direction de L. Schwartz.
\end{abstract}

AMS Classification $55 \mathrm{~N} 99 ; 55 \mathrm{~S} 10$

Keywords Opérations de Steenrod; module instable; théorème d'Adams; la classification des modules instables injectifs

\section{Introduction}

En topologie algébrique, pour distinguer les espaces, on introduit des invariants, tels que l'homologie, la cohomologie et les groupes d'homotopie des espaces. 
Nous nous intéressons dans cet article à la cohomologie réduite des espaces en tant que module instable sur l'algèbre de Steenrod. Nous considérons d'abord le cas $p=2$, les généralisations pour les nombres $p$ premiers impairs seront données dans la dernière section.

Un problème central sur les modules instables est de savoir quand un tel module est la cohomologie réduite d'un espace. Un résultat célèbre de J.F. Adams impose des restrictions fortes à un module instable pour qu'il soit la cohomologie réduite d'un espace. Voici le résultat d'Adams dont il est question:

Théorème 1 (Adams [1]) Soit $X$ un espace ou un spectre, $k \geq 4$, soit $x \in H^{n}(X ; \mathbb{Z} / 2)$ tel que $S q^{2^{i}} x=0, \forall i<k$, alors $S q^{2^{k}} x \in \sum_{i<k} \operatorname{Im}\left(S q^{2^{i}}\right)$.

Définition 1 Un module sur l'algèbre de Steenrod $M$ est un module instable si pour tout élément $x \in M, S q^{i} x=0$ quand $i>|x|$. Ici, $|x|$ désigne le degré de $x$.

Comme $S q^{0}$ est l'identité, ceci implique que les modules instables sont triviaux en degré strictement inférieur à zéro.

Définition 2 Par lacune de longueur $d$ dans un module instable $M$, on entend une suite d'entiers $I=\{i, \cdots, i+d-1\}$ telle que $M^{j}=\{0\}$, si $j \in I, M^{i-1} \neq$ $\{0\}, M^{i+d} \neq\{0\}$. On note cette lacune par $(i-1, i+d)$ ou $(i-1, i+d-1]$.

Issue du théorème d'Adams, une question intéressante est de savoir si dans la cohomologie mod 2 d'un espace, il peut exister ou non des lacunes de longueur arbitrairement grande. Dans cet article, on démontre que c'est impossible sous certaines hypothèses supplémentaires sur la structure du module instable.

Nous devons rappeler, pour énoncer ces conditions, diverses définitions. Rappelons qu'un module $M$ est connexe si $M^{\leq 0}=\{0\}$, un module instable est donc connexe si $M^{0}=\{0\}$.

Définition 3 La suspension d'un module instable $M$ est le module $\Sigma M$ tel que $(\Sigma M)^{n}=M^{n-1}, \forall n$.

Définition 4 Un module instable $M$ est réduit si le morphisme $S q_{0}: M \rightarrow M$ défini par $S q_{0}(x)=S q^{|x|}(x), \forall x \in M$, est injectif. 
On va se restreindre dans la suite à étudier des modules instables dont l'enveloppe injective est somme directe finie d'objets injectifs indécomposables. D'après la classification des $\mathcal{U}$-injectifs (Lannes-Schwartz, [5]), on sait que pour un tel module instable réduit $M$, il existe des entiers $d$ et $\alpha_{d}$ tels que $M$ se plonge dans $H^{*}\left(B(\mathbb{Z} / 2)^{\oplus d} ; \mathbb{Z} / 2\right)^{\oplus \alpha_{d}}$. Si $M$ est connexe, on peut supposer $\alpha_{d}=1$. Donc pour établir une propriété pour les modules instables réduits, il suffit de le faire pour les sous-modules instables de $H^{*}\left(B(\mathbb{Z} / 2)^{\oplus d} ; \mathbb{Z} / 2\right)^{\oplus \alpha_{d}}$. Dans la suite on supposera $\alpha_{d}=1$, les démonstrations s'étendent sans problème.

Définition 5 (Schwartz 9]) Un module instable $M$ est $s$-nilpotent s'il est l'union de ses sous-modules ayant une filtration finie dont les quotients sont des $s$-ème suspensions.

Soit $\mathcal{U}$ la catégorie des modules instables. On désigne $\mathcal{N} i l_{s}$ la sous-catégorie abélienne pleine de $\mathcal{U}$ des modules $s$-nilpotents. La sous-catégorie $\mathcal{N} i l_{s}$ est épaisse (voir 2], 10]). On a une filtration de $\mathcal{U}$ :

$$
\cdots \subset \mathcal{N} i l_{2} \subset \mathcal{N} i l_{1}=\mathcal{N} i l \subset \mathcal{N} i l_{0}=\mathcal{U} .
$$

Soit $n i l_{s}: \mathcal{U} \rightarrow \mathcal{N} i l_{s}$ l'adjoint à droite de l'inclusion $\mathcal{N} i l_{s} \hookrightarrow \mathcal{U}, n i l_{s} M$ est le plus grand sous-module d'un module instable $M$ dans $\mathcal{N} i l_{s}$ et on a la filtration nilpotente de $M$ :

$$
\cdots \subset \operatorname{nil}_{2} M \subset \operatorname{nil}_{1} M \subset n i l_{0} M=M .
$$

Proposition 1 (4], 8]) Soit $M$ un module instable. Alors le quotient $n i l_{s} M / n i l_{s+1} M$ est la $s$-ème suspension d'un module instable réduit $R_{s}$, donc

$$
n i l_{s} M / n i l_{s+1} M \cong \Sigma^{s} R_{s} .
$$

Définition 6 La filtration nilpotente d'un module instable $M$ est de longueur finie s'il existe un $n \geq 0$ tel que $n i l_{n} M=0$.

Définition 7 Soit $M$ un module instable connexe réduit non-trivial. On désigne par $n_{1}<n_{2}<\cdots$ les degrés $n$ tels que $M^{n} \neq\{0\}$. Supposons que $M$ se plonge dans $H^{*}\left(B(\mathbb{Z} / 2)^{\oplus d} ; \mathbb{Z} / 2\right)$. Le module instable $M$ sera dit de type $\mathcal{T}$, s'il contient une lacune $(s, s+l]$ avec $s \geq n_{1}$ et

$$
l \geq \max \left\{2^{d+4}, n_{j+1}-n_{j} \mid j=1, \cdots, 1+(d-1) 2^{d-2}\right\} .
$$

Remarque Le module $M$ est nécessairement infini car $M$ est réduit nontrivial. 
Le résultat principal de cet article est le théorème suivant:

Théorème 2 Soit $M$ un $\mathcal{A}_{2}$-module qui est une suspension itérée d'un sousmodule de type $\mathcal{T}$ de $\tilde{H}^{*}\left(B(\mathbb{Z} / 2)^{\oplus d} ; \mathbb{Z} / 2\right)$. Alors $M$ n'est pas réalisable, i.e., il n'existe aucun espace $X$ tel que $M=\tilde{H}^{*}(X ; \mathbb{Z} / 2)$.

En fait le théorème d'Adams s'applique aussi aux spectres. Il en est donc de même du théorème précédent, la suspension itérée peut être positive ou négative et le module n'est ni la cohomologie réduite d'un espace ni la cohomologie d'un spectre.

Une généralisation de ce théorème est faite sous certaines hypothèses pour les modules instables connexes ayant une filtration nilpotente de longueur finie.

Définition 8 Soit $M$ un module instable infini connexe dont la filtration nilpotente est de longueur finie. Les quotients ${ }_{i l} l_{s} M / n i l_{s+1} M$ non-triviaux s'écrivent sous la forme $\Sigma^{m_{i}} R_{m_{i}}, R_{m_{i}}$ réduits, $i=1, \cdots, t, m_{1}<\cdots<m_{t}$. Notons que l'un au moins des $R_{m_{i}}$ est infini. Supposons qu'il existe des entiers $d$ et $\alpha_{d}$ tels que tous les $R_{m_{i}}$ se plongent dans $H^{*}\left(B(\mathbb{Z} / 2)^{\oplus d} ; \mathbb{Z} / 2\right)^{\oplus \alpha_{d}}$. Notons $I \subset\{1, \cdots, t\}$ le sous-ensemble des $i$ tels que $R_{m_{i}}$ soit infini, et soit $n_{1, i}<$ $n_{2, i}<\cdots$ les degrés en lesquels ce module est non-trivial.

Soit $\delta$ tel que $2^{\delta} \geq t>2^{\delta-1}$. Le module instable $M$ sera dit de type $\mathcal{T}$ s'il contient une lacune $(s, s+l]$ avec $s \geq \min \left\{m_{i}+n_{1, i} \mid i \in I\right\}$ et

$$
l \geq \max \left\{\left(m_{t}+1\right) 2^{d+4}, n_{j+1, i}-n_{j, i} \mid i \in I, j=1, \cdots, 1+(d+\delta-1) 2^{d-2}\right\} .
$$

Condition 1 Soit $M$ un module instable connexe dont la filtration nilpotente est de longueur finie. En utilisant les notations introduites dans la définition précédente, on dira que $M$ vérifie la condition 1 si

$$
\begin{array}{cl}
m_{i+1}-m_{i} \neq 1,2,4,8, & 1 \leq i \leq t-1, \\
m_{i+2}-m_{i} \neq 8, & 1 \leq i \leq t-2,
\end{array}
$$

c'est-à-dire, $m_{j}-m_{i}=2^{\beta}$ n'a pas de solution pour $1 \leq i, j \leq t$ et $0 \leq \beta \leq 3$.

Théorème 3 Soit $M$ un module qui est une suspension itérée (positive ou négative) d'un module instable connexe dont la filtration nilpotente est de longueur finie, qui est de type $\mathcal{T}$ et vérifie la condition [1. Alors $M$ n'est pas réalisable, i.e., il n'existe aucun espace ou spectre $X$ tel que $M=\tilde{H}^{*}(X ; \mathbb{Z} / 2)$.

Corollaire 1 La longueur des lacunes ne peut pas être arbitrairement grande dans un module instable connexe réalisable dont la filtration nilpotente est de longueur finie et qui vérifie la condition [1] 
Dans cet article, on ne considère que les modules dont l'enveloppe injective est somme directe finie de modules injectifs indécomposables. Les résultats obtenus sont conséquences du théorème d'Adams et de la classification de Lannes-Schwartz.

Voici quelques détails sur le plan de cet article. Dans la section 2, on définit des opérations $Q_{t}^{s}, s, t \geq 0$, qui généralisent les opérations de Milnor. La section 3 contient un résultat combinatoire. En utilisant ce résultat, le théorème 2 est démontré dans la section 4 . Ensuite, le théorème 3 est démontré dans la section 5. La dernière section contient des généralisations pour le cas $p$ premier impair. Il y a un appendice à la fin sur les opérations $Q_{t}^{s}$.

L'auteur tient à remercier le rapporteur pour ses remarques et ses conseils, qui l'ont aidé à éviter bien des imprécisions dans les définitions et démonstrations.

\section{Les opérations $Q_{t}^{s}, s, t \geq 0$}

Dans cette section, on définit les opérations $Q_{t}^{s}, s, t \geq 0$ et on donne brièvement leurs propriétés utilisées dans les sections suivantes. Pour plus de détails sur ces opérations, on renvoie le lecteur à l'appendice.

Définition 9 Les opérations $Q_{t}^{s}, s, t \geq 0$ sont définies récursivement comme suit:

(1) $Q_{0}^{s}=S q^{2^{s}}$;

(2) $Q_{t+1}^{s}=\left[S q^{2^{s+t+1}}, Q_{t}^{s}\right]$.

Notation 1 On note souvent $Q_{t}^{0}$ par $Q_{t}$, qui est la notation usuelle de l'opération de Milnor concernée [7].

Pour établir les propriétés de ces opérations $Q_{t}^{s}$, on a besoin d'introduire quelques notations.

Notation 2 Le symbole $\left(n_{1}, \cdots, n_{d}\right)$ désignera le monôme $u^{n_{1}} \otimes \cdots \otimes u^{n_{d}}$ ou $x_{1}^{n_{1}} \cdots x_{d}^{n_{d}}$ dans $H^{*}\left(B(\mathbb{Z} / 2)^{\oplus d} ; \mathbb{Z} / 2\right)$ qui s'identifie à $\mathbb{F}_{2}[u]^{\otimes d}$ ou $\mathbb{F}_{2}\left[x_{1}, \cdots, x_{d}\right]$, $u$ et les $x_{i}$ étant de degré 1 . Un tel monôme sera dit basique.

Notation 3 Comme plus haut, $S q_{0}$ désigne l'opération définie dans un module instable par $S q_{0} x=S q^{|x|} x$. On a donc $S q_{0}^{s}\left(n_{1}, \cdots, n_{d}\right)=\left(2^{s} n_{1}, \cdots, 2^{s} n_{d}\right)$, et $\operatorname{Im}\left(S q_{0}^{s}\right)$ est l'ensemble des éléments $x=\sum_{i \in I}\left(2^{s} n(i)_{1}, \cdots, 2^{s} n(i)_{d}\right)$ de $H^{*}\left(B(\mathbb{Z} / 2)^{\oplus d} ; \mathbb{Z} / 2\right)$. Ici, $I$ est un ensemble d'indices $i$ qui indexent des différents $d$-uplets $\left(n(i)_{1}, \cdots, n(i)_{d}\right), n(i)_{\alpha}$ peut être nul. 
Lemme 1 Soit $M$ un module instable, on a pour tout $n \geq 1$,

$$
S q^{2 n} S q_{0} x=S q_{0} S q^{n} x, \quad \forall x \in M .
$$

De la définition de $Q_{t}^{s}$ et de $S q_{0}$, on déduit que:

Lemme 2 Soit $M$ un module instable, on a $\forall x \in M$,

$$
Q_{t}^{s+r} S q_{0}^{s} x=S q_{0}^{s} Q_{t}^{r} x, \quad \forall r, s, t .
$$

En particulier,

$$
Q_{t}^{s} S q_{0}^{s} x=S q_{0}^{s} Q_{t} x, \quad \forall s, t .
$$

Corollaire 2 Soient $M, N$ deux modules instables, et soient $l, r, s, t \geq 0$.

(1) $\forall x \in M, Q_{r}^{s} Q_{t}^{s} S q_{0}^{s} x=Q_{t}^{s} Q_{r}^{s} S q_{0}^{s} x$ et $\left(Q_{t}^{s}\right)^{2} S q_{0}^{s} x=0$.

(2) $\forall x \in S q_{0}^{s}(M)$ et $y \in S q_{0}^{s}(N), Q_{t}^{s}(x \otimes y)=Q_{t}^{s} x \otimes y+x \otimes Q_{t}^{s} y$.

(3) Soit u le générateur de $H^{*}(B(\mathbb{Z} / 2) ; \mathbb{Z} / 2)$ en degré 1 , $Q_{t}^{s} S q_{0}^{s} u^{2 l}=0$ et $Q_{t}^{s} S q_{0}^{s} u^{2 l+1}=S q_{0}^{s} u^{2 l+2^{t+1}}=u^{2^{s}\left(2 l+2^{t+1}\right)}$.

Le lemme 1 est une conséquence directe de la définition de $S q_{0}$, sa démonstration est laissée au lecteur. Pour le lemme 2 et le corollaire 2] leurs démonstrations se trouvent dans l'appendice.

\section{Un résultat combinatoire}

Dans cette section, on établit d'abord un résultat combinatoire. Ensuite, on l'applique à un élément quelconque de $H^{*}\left(B(\mathbb{Z} / 2)^{\oplus d} ; \mathbb{Z} / 2\right)$ pour obtenir des contraintes imposées par certaines conditions d'annulation induites par l'existence de lacunes.

Soit un élément $x \in H^{*}\left(B(\mathbb{Z} / 2)^{\oplus d} ; \mathbb{Z} / 2\right), \quad x=\sum_{i \in I}\left(n(i)_{1}, \cdots, n(i)_{d}\right)$ est somme de monômes basiques deux à deux distincts $\left(n(i)_{1}, \cdots, n(i)_{d}\right), i \in I$.

Pour commencer, on définit quelques notations combinatoires.

Définition 10 Soit $g \geq 0$, on dira qu'il y a un $g$-échange entre deux monômes basiques $\alpha$ et $\beta$ s'il existe $i$ et $j$ tels que ces deux monômes constituent, à un ordre (entre $i$ et $j$ ) près, une paire de la forme

$$
\begin{aligned}
\alpha & =\left(u_{1}, \cdots, u_{i-1}, 2 u_{i}+1, u_{i+1}, \cdots, u_{j-1}, 2 u_{j}+2^{g+1}, u_{j+1}, \cdots, u_{d}\right) \\
\text { et } \quad \beta & =\left(u_{1}, \cdots, u_{i-1}, 2 u_{i}+2^{g+1}, u_{i+1}, \cdots, u_{j-1}, 2 u_{j}+1, u_{j+1}, \cdots, u_{d}\right) .
\end{aligned}
$$

On dira plus précisément, s'il y a lieu, qu'il y a un $g$-échange en $i$-ème position pour le monôme $\alpha$ avec le monôme $\beta$. 
Remarque C'est l'annulation sous l'action de l'opération de Milnor $Q_{g}$ sur un élément $x$ qui suggère cette définition, puisque $Q_{g}$ est une dérivation.

Définition 11 On dira qu'il y a une $(l, s)$-chaîne, $l \leq s$, entre deux monômes basiques $\alpha$ et $\beta$ d'un sous-ensemble de l'ensemble des monômes basiques d'un élément $x \in H^{*}\left(B(\mathbb{Z} / 2)^{\oplus d} ; \mathbb{Z} / 2\right)$ s'il existe des monômes basiques:

$$
\alpha=\alpha_{0}, \alpha_{1}, \cdots, \alpha_{t}=\beta
$$

dans ce sous-ensemble tels qu'il y ait un $m$-échange, $l \leq m \leq s$, entre $\alpha_{i}$ et $\alpha_{i+1}$ pour tout $i=0, \cdots, t-1$.

Définition 12 Soit $x \in H^{*}\left(B(\mathbb{Z} / 2)^{\oplus d} ; \mathbb{Z} / 2\right)$. On dira qu'un sous-ensemble $S$ de l'ensemble des monômes basiques de $x$ admet $T \subset\{1, \cdots, d\}$ pour support, si pour tout monôme basique $x_{1}^{\alpha_{1}} \cdots x_{d}^{\alpha_{d}}$ appartenant à $S$ et pour tout $i \in T$, l'exposant $\alpha_{i}$ ne dépend que de $S$ et pas du mônome basique choisi et est de plus pair. On suppose de plus $T$ maximal parmi les sous-ensembles de $\{1, \cdots, d\}$ ayant cette propriété.

On note $\tau=\# T$ que l'on appellera la taille de $T$, les monômes basiques de $S$ ont donc $\tau$ exposants en commun et s'écrivent tous sous la forme $y^{2} z$ où $y$ dépend de $\tau$ variables $x_{i}$ et ne dépend pas du monôme basique choisi; $z$ dépend lui de $d-\tau$ variables et du monôme basique choisi.

Définition 13 Un sous-ensemble de l'ensemble des monômes basiques d'un élément $x \in H^{*}\left(B(\mathbb{Z} / 2)^{\oplus d} ; \mathbb{Z} / 2\right)$ est appelé une $(l, s)$-classe, $l \leq s$, de support $T \subset\{1, \cdots, d\}$, si la condition suivante a lieu: pour tout monôme basique $\alpha$ dans ce sous-ensemble, il existe au moins une position $i$ dont l'exposant est impair; pour toutes ces positions $i$ et tous les $m, l \leq m \leq s$, il existe un monôme $\beta$ dans le sous-ensemble et un $m$-échange en $i$-ème position pour $\alpha$ avec $\beta$.

Remarque C'est l'annulation sous l'action des opérations $Q_{m}, l \leq m \leq s$, sur un élément $x$ qui suggère cette définition, puisque les opérations $Q_{m}$ sont des dérivations.

Voici la propriété fondamentale des $(l, s)$-classes de support $T$ :

Proposition 2 Pour toute $(l, s)$-classe de support $T$ d'un élément $x \in$ $H^{*}\left(B(\mathbb{Z} / 2)^{\oplus d} ; \mathbb{Z} / 2\right)$, on a $s-l+\# T \leq d-2$. 
Démonstration Considérons une $(l, s)$-classe admettant $T=T_{s}$ pour support, soit $\tau_{s}$ sa taille. Pour $1 \leq t \leq s-l$, on va construire récursivement des $(l, s-t)$-classes de support $T_{s-t}$ de taille $\tau_{s-t}$ telles que

$$
\tau_{s-t} \geq \tau_{s-t+1}+1 \text {. }
$$

Pour $t=s-l$, on aura une $(l, l)$-classe de support $T_{l}$ dont la taille $\tau_{l}$ sera telle que $\tau_{l} \geq \tau_{l+1}+1 \geq \cdots \geq \tau_{s}+s-l=\# T+s-l$. Comme cette classe comporte des $l$-échanges, on a $\tau_{l} \leq d-2$. D'où,

$$
d-2 \geq \tau_{l} \geq \# T+s-l .
$$

Supposons avoir construit une $(l, s-t+1)$-classe de support $T_{s-t+1}$ de taille $\tau_{s-t+1}$. On va construire une sous $-(l, s-t)$-classe, de la $(l, s-t+1)$-classe initiale, dont le support sera obtenu par adjonction à $T_{s-t+1}$ d'une position où l'exposant d'un certain monôme $\beta$ prend une valeur paire.

On considère parmi les exposants impairs qui apparaissent dans les monômes basiques de la $(l, s-t+1)$-classe la valeur maximale, soit $2 a+1$. Notons qu'il apparait nécessairement des exposants impairs car il y a des $m$-échanges, $l \leq m \leq s-t+1$. On suppose que cet exposant apparait en position $p$ d'un monôme basique $\alpha$ de la $(l, s-t+1)$-classe. Soit alors $\beta$ un monôme dans la $(l, s-t+1)$-classe tel qu'il existe un $(s-t+1)$-échange en position $p$ pour $\alpha$ avec $\beta$. Le monôme $\beta$ existe par hypothèse.

Si on désigne par $\psi_{p}$, l'exposant en position $p$ d'un monôme basique $\psi$, on a alors $\alpha_{p}=2 a+1$ et $\beta_{p}=2 a+2^{s-t+2}$.

Lemme 3 Pour tout monôme basique $\gamma$ d'une $(l, s-t)$-chaîne contenue dans la $(l, s-t+1)$-classe et contenant $\beta$ la valeur $\gamma_{p}$ de l'exposant en position $p$ est $2 a+2^{s-t+2}$.

Démonstration Raisonnons par l'absurde et choisissons une $(l, s-t)$-chaîne contenue dans la $(l, s-t+1)$-classe qui ne satisfasse pas à cette condition et soit de longueur minimale. Soit $\beta=\beta_{0}, \cdots, \beta_{u}=\gamma$ cette chaîne. L'exposant $\gamma_{p}$ est impair. Il y a un $m$-échange, $l \leq m \leq s-t$, entre $\beta_{u-1}$ et $\gamma$ en position $p$. Mais $\left(\beta_{u-1}\right)_{p}=2 a+2^{s-t+2}$, donc $\gamma_{p}=2 a+2^{s-t+2}-2^{m+1}+1>2 a+1$, en contradiction avec la maximalité de $2 a+1$.

Considérons alors l'ensemble $S$ des monômes basiques de la $(l, s-t+1)$-classe tels qu'il existe une $(l, s-t)$-chaîne entre ces monômes et $\beta$. 
Lemme 4 L'ensemble $S$ est une $(l, s-t)$-classe de support contenant $T_{s-t+1} \cup$ $\{p\}$.

Démonstration Comme chaque monôme basique de l'ensemble $S$ est par définition un monôme basique de la $(l, s-t+1)$-classe, il contient donc au moins un exposant impair. Pour montrer que $S$ est une $(l, s-t)$-classe, il faut encore montrer qu'en toute position $q$ où un des monômes de cet ensemble a un exposant impair, il y a pour tout $m, l \leq m \leq s-t$, un $m$-échange en position $q$ pour chacun de ces monômes avec un autre monôme dans $S$. Mais un tel monôme existe par hypothèse dans la $(l, s-t+1)$-classe et ce monôme est alors par définition dans $S$ puisqu'il y a une $(l, s-t)$-chaîne à $\beta$. Clairement le suport $T_{s-t}$ contient $T_{s-t+1} \cup\{p\}$.

Il reste à observer pourquoi on peut mener le processus jusqu'à $t=s-l$, car dans cette construction comme il y a des $m$-échanges, $l \leq m \leq s-t$, il y a des exposants impairs.

\section{Fin de la démonstration de la proposition}

Corollaire 3 Soit $x$ un élément de $H^{*}\left(B(\mathbb{Z} / 2)^{\oplus d} ; \mathbb{Z} / 2\right)$ tel que $x \in \operatorname{Im}\left(S q_{0}^{s}\right)$ $\operatorname{Im}\left(S q_{0}^{s+1}\right)$ et que $Q_{t}^{s} x=0, \forall p \leq t \leq q$. Alors on a $q-p \leq d-2$.

Démonstration Puisque $x=S q_{0}^{s} x^{\prime} \in \operatorname{Im}\left(S q_{0}^{s}\right)-\operatorname{Im}\left(S q_{0}^{s+1}\right)$, il existe au moins un monôme basique $\alpha$ de $x^{\prime}$ avec au moins un exposant impair. L'ensemble des monômes basiques de $x^{\prime}$ qui sont dans une $(p, q)$-chaîne contenant $\alpha$ est une $(p, q)$-classe dont on note le support par $T$. Précisons un peu. D'après la définition, chaque monôme basique $\beta$ de cet ensemble contient au moins un exposant impair (à cause de l'existence d'un échange avec un autre monôme basique de l'ensemble). On note par $I_{\beta}$ l'ensemble non vide des positions des exposants impairs dans $\beta$. Comme l'action de l'opération $Q_{t}$ sur $\beta$ donne un monôme qui contient un exposant pair en la même position, l'annulation de $Q_{t}^{s}$ sur $x$ entraîne l'existence d'un $m$-échange, $p \leq m \leq q$, en $i$-ème position, $i \in I_{\beta}$, pour $\beta$ avec un monôme basique de l'ensemble.

Par conséquent, la proposition 2 nous donne $q-p \leq q-p+\# T \leq d-2$.

Corollaire 4 Soit $x$ un élément de $H^{*}\left(B(\mathbb{Z} / 2)^{\oplus d} ; \mathbb{Z} / 2\right)$. Si $\mathcal{A}_{2} x$ contient une lacune $(|x|,|x|+l], l \geq 2^{k}$ pour un certain $k \geq d-2$, alors $x \in \operatorname{Im}\left(S q_{0}^{k-d+2}\right)$. 
Démonstration Soit $\alpha$ tel que $x=S q_{0}^{\alpha} x^{\prime} \in \operatorname{Im}\left(S q_{0}^{\alpha}\right)-\operatorname{Im}\left(S q_{0}^{\alpha+1}\right)$. Si $\alpha>k$, on a $\alpha \geq k-d+2$. Si $\alpha \leq k$, alors l'existence de la lacune dans $\mathcal{A}_{2} x$ implique que $\forall t=0, \cdots, k-\alpha$,

$$
S q_{0}^{\alpha} S q^{2^{t}} x^{\prime}=S q^{2^{t+\alpha}} S q_{0}^{\alpha} x^{\prime}=S q^{2^{t+\alpha}} x=0 .
$$

Donc $S q^{2^{t}} x^{\prime}=0, \forall t=0, \cdots, k-\alpha$. Donc $Q_{t} x^{\prime}=0, \forall t=0, \cdots, k-\alpha$. D'après le corollaire [3] on a $k-\alpha \leq d-2$, d'où $\alpha \geq k-d+2$.

Remarques (1) Les énoncés de cette section sont aussi vrais pour un élément quelconque de $H^{*}\left(B(\mathbb{Z} / 2)^{\oplus d} ; \mathbb{Z} / 2\right)^{\oplus \alpha_{d}}$. Ci-dessus, on a traité le cas où $\alpha_{d}=1$. Pour tenir compte $d u$ fait que l'on peut se placer dans $H^{*}\left(B(\mathbb{Z} / 2)^{\oplus d} ; \mathbb{Z} / 2\right)^{\oplus \alpha_{d}}$, il faudrait compliquer un peu les notations en rajoutant un indice $1 \leq a \leq \alpha_{d}$. On dit que les $\left(n(i)_{1, a}, \cdots, n(i)_{d, a}\right)$ sont les monômes basiques de $x$.

(2) Comme la suspension commute avec les opérations de Steenrod ( $\sigma^{q} S q^{i}=$ $S q^{i} \sigma^{q}$ ), on peut aussi établir les énoncés similaires de ces corollaires pour une suspension quelconque de $H^{*}\left(B(\mathbb{Z} / 2)^{\oplus d} ; \mathbb{Z} / 2\right)^{\oplus \alpha_{d}}$.

\section{Démonstration du théorème 2}

Cette section est consacrée à la démonstration du théorème 2. Soit donc $M$ un module instable qui est la cohomologie réduite d'un espace ou d'un spectre. Supposons de plus que $M$ est réduit. Alors:

Théorème 4 (Lannes-Schwartz [5]) Un module instable réduit (resp. réduit et connexe) $M$ dont l'enveloppe injective est somme directe finie d'injectifs indécomposables est isomorphe à un sous-module de $H^{*}\left(B(\mathbb{Z} / 2)^{\oplus d} ; \mathbb{Z} / 2\right)^{\oplus \alpha_{d}}$ $\left(\alpha_{d}>0\right)\left(\right.$ resp. $\left.H^{*}\left(B(\mathbb{Z} / 2)^{\oplus d} ; \mathbb{Z} / 2\right)\right)$ pour $d$ assez grand.

Démonstration du Théorème 2 Dans la suite, on va démontrer l'énoncé suivant: Soit $M$ un sous-module de type $\mathcal{T}$ de $\tilde{H}^{*}\left(B(\mathbb{Z} / 2)^{\oplus d} ; \mathbb{Z} / 2\right)$. Alors $M$ n'est pas réalisable, i.e., il n'existe aucun espace ou spectre $X$ tel que $M=$ $\tilde{H}^{*}(X ; \mathbb{Z} / 2)$. On note que, une fois cet énoncé est établi, le théorème 2 est aussi établi.

On raisonne par l'absurde. Soit $M$ un sous-module de type $\mathcal{T}$ de $\tilde{H}^{*}\left(B(\mathbb{Z} / 2)^{\oplus d} ; \mathbb{Z} / 2\right)$ qui est la cohomologie réduite d'un espace ou d'un spectre. Reprenons les notations introduites avant le théorème 2 $M$ est non-trivial 
dans les degrés $n_{1}<n_{2}<\cdots$. Supposons que pour $n \geq n_{1},(n, n+l]$ soit la première lacune de longueur $l$ telle que:

$$
l \geq \max \left\{2^{d+4}, n_{j+1}-n_{j} \mid j=1, \cdots, 1+(d-1) 2^{d-2}\right\} .
$$

Soit $k$ l'unique entier $(\geq d+4)$ tel que $2^{k+1}>l \geq 2^{k}$.

Soit donc $x \in M^{n}, x \neq 0$. Alors $\mathcal{A}_{2} x$ contient une lacune $(n, n+l]$ et le corollaire 4 entraîne $2^{k-d+2} \mid n$.

Lemme 5 En degré strictement inférieur à $n$, il n'existe pas de degrés $m$ tels que $2^{k-d+2} \not$ rm et $M^{m} \neq\{0\}$.

Démonstration Supposons qu'en degré strictement inférieur à $n$, il existe des degrés $m$ tels que $2^{k-d+2} \not m$ et $M^{m} \neq\{0\}$. Soit $m_{0}$ le plus grand de ces degrés, et soit $y \in M^{m_{0}}, y \neq 0$. On suppose que $y=S q_{0}^{\alpha} z \in \operatorname{Im}\left(S q_{0}^{\alpha}\right)-\operatorname{Im}\left(S q_{0}^{\alpha+1}\right)$. Comme $2^{k-d+2} \not m_{0}$, on a $\alpha \leq k-d+1$.

Si $\mathcal{A}_{2} y$ contient une lacune $(|y|, n+l]$, le corollaire 4 implique que $\alpha \geq k-d+2$, ce qui est impossible. Donc le plus bas degré supérieur ou égal à $m_{0}+1$, en lequel $\mathcal{A}_{2} y$ est non-trivial, est inférieur à $n+l$ et est donc de la forme $2^{k-d+2} q=: p$ d'après l'hypothèse de maximalité de $m_{0}$.

Comme les $S q^{2^{h}}$ engendrent multiplicativement $\mathcal{A}_{2}$, on a $p-m_{0}=: 2^{\beta}$ (rappelons l'hypothèse de minimalité de $p$ ). En particulier, $S q^{2^{\beta}} y$ est non nul. Comme $y \in \operatorname{Im}\left(S q_{0}^{\alpha}\right)$, on a alors $\beta \geq \alpha$ car $S q^{2^{\beta}} S q_{0}^{\alpha} z=S q_{0}^{\alpha} S q^{2^{\beta-\alpha}} z$ qui est nul si $\alpha>\beta$. On va montrer que $\alpha \geq k-d$.

Supposons $\alpha \leq k-d$ et $t \geq 1$. Alors,

Lemme $6 Q_{t}^{\alpha} y$ est nul, tant que son degré est inférieur ou égal à $n+l$.

Démonstration Par l'hypothèse de maximalité de $m_{0}$, on sait qu'il suffit de montrer que $2^{k-d+2}$ ne divise pas le degré de $Q_{t}^{\alpha} y$. En effet on a:

$$
\begin{aligned}
\left|Q_{t}^{\alpha} y\right| & =m_{0}+2^{\alpha}\left(2^{t+1}-1\right) \\
& =2^{k-d+2} q-2^{\beta}+2^{\alpha+t+1}-2^{\alpha} \\
& =2^{k-d+2} q+\left(2^{\alpha+t}+\cdots+2^{\alpha+1}+2^{\alpha}\right)-2^{\beta} .
\end{aligned}
$$

Comme $t \geq 1$ et $\beta \geq \alpha$, on sait que ce degré est un multiple impair de $2^{\alpha}$ pour $\beta>\alpha$ et que c'est un multiple impair de $2^{\alpha+1}$ pour $\beta=\alpha$. Donc $2^{\alpha+2}$ ne divise pas ce degré. Puisque $\alpha+2 \leq k-d+2,2^{k-d+2}$ ne divise pas ce degré non plus. 
Or pour $1 \leq t \leq k-\alpha-1$,

$$
\begin{aligned}
\left|Q_{t}^{\alpha} y\right| & =m_{0}+2^{\alpha}\left(2^{t+1}-1\right) \\
& \leq m_{0}+2^{\alpha}\left(2^{k-\alpha}-1\right) \\
& \leq n+l
\end{aligned}
$$

Donc d'après le corollaire 3 $k-\alpha-2 \leq d-2$, alors $\alpha \geq k-d$ et par conséquent, $\beta \geq \alpha \geq k-d$.

D'après la définition de $\beta, p=m_{0}+2^{\beta}$, et par hypothèse, $2^{k-d+2} \not m_{0}$ et $2^{k-d+2} \mid p$, on a donc $\beta \leq k-d+1$, ce qui implique que $M$ contient une lacune $\left(m_{0}, p\right)$. Car sinon, il existe $p^{\prime} \in\left(m_{0}, p\right)$ tel que $M^{p^{\prime}} \neq\{0\}$. Par l'hypothèse de maximalité de $m_{0}, 2^{k-d+2} \mid p^{\prime}$. Donc $2^{\beta}=p-m_{0}>p-p^{\prime} \geq 2^{k-d+2}$, ce qui est contradictoire au fait que $\beta \leq k-d+1$. L'existence de la lacune $\left(m_{0}, p\right)$ dans $M$ et le théorème d'Adams impliquent que $\beta \leq 3$. Or comme $\beta \geq k-d$ et $k \geq d+4$, on a $\beta \geq k-d \geq 4$, ceci implique qu'un tel $m_{0}$ n'existe pas.

\section{Fin de la démonstration du Lemme 5}

Notons donc les degrés plus petits que $n$ pour lesquels $M$ est non-trivial comme suit

$$
n=r_{0}>r_{1}>\cdots \quad \text { et } \forall i, 2^{k-d+2} \mid r_{i} .
$$

On a

Lemme 7 Pour tout $x_{i}$ de degré $r_{i}, \mathcal{A}_{2} x_{i}$ contient la lacune $\left(r_{i}, n+l\right]$.

Démonstration On raisonne par l'absurde. Si l'énoncé est faux, on choisit un élément $x_{i}$ de degré maximal tel que $\mathcal{A}_{2} x_{i}$ contienne des éléments non nuls de degré supérieur à $r_{i}$ et inférieur à $n+l$. On choisit dans $\left(r_{i}, n+l\right]$ le plus bas degré en lequel $\mathcal{A}_{2} x_{i}$ est non-trivial. En utilisant la base multiplicative de $\mathcal{A}_{2}$, il est de la forme $r_{i}+2^{\gamma}, \gamma \geq 0$. Comme les degrés dans l'intervalle $\left(r_{i}, n+l\right]$ où il y a des éléments non nuls sont divisibles par $2^{k-d+2}$, on a $\gamma \geq k-d+2 \geq 6$. Par conséquent, on a une lacune $\left(r_{i}, r_{i}+2^{\gamma}\right), \gamma \geq 6$, dans $\mathcal{A}_{2} x_{i}$ avec $S q^{2^{\gamma}} x_{i} \neq 0$ en degré $r_{i}+2^{\gamma}$. Par la maximalité de $x_{i}$, il n'y a aucun élément $y$ de degré supérieur à $r_{i}$ tel que $\mathcal{A}_{2} y$ contienne des éléments non nuls en degré supérieur à $|y|$ et inférieur à $n+l$. Donc l'élément non nul $S q^{2^{\gamma}} x_{i}$ ne peut pas être dans $\sum_{j<\gamma} \operatorname{Im}\left(S q^{2^{j}}\right)$. Alors l'existence de cette lacune $\left(r_{i}, r_{i}+2^{\gamma}\right)$ dans $\mathcal{A}_{2} x_{i}$ est impossible à cause du théorème d'Adams.

On montre alors par récurrence que:

$$
\forall i \geq j 2^{d-2}, \quad 2^{k-d+j+2} \mid r_{i} .
$$


Le cas $j=0$ est démontré ci-dessus. Supposons que c'est vrai pour $j$, alors

$$
\begin{aligned}
& n+l-r_{(j+1) 2^{d-2}}=l+\sum_{i=0}^{(j+1) 2^{d-2}-1}\left(r_{i}-r_{i+1}\right) \\
& =l+\sum_{h=0}^{j} \sum_{i=h 2^{d-2}}^{(h+1) 2^{d-2}-1}\left(r_{i}-r_{i+1}\right) \\
& \geq 2^{k}+\sum_{h=0}^{j} 2^{d-2} 2^{k-d+h+2} \\
& =2^{k}+\sum_{h=0}^{j} 2^{k+h} \\
& =2^{k+j+1} \text {. }
\end{aligned}
$$

Puisque $\mathcal{A}_{2} x_{i}$ contient une lacune $\left(r_{i}, n+l\right]$ et d'après le corollaire 4, on a donc $2^{k-d+j+3} \mid r_{i}, \forall i \geq(j+1) 2^{d-2}$.

Rappelons que par l'hypothèse sur $l$, il y a bien (au moins) $(d-1) 2^{d-2}+1$ valeurs pour l'indice $i$ de $r_{i}$. On peut donc poser $w=(d-1) 2^{d-2}$, alors $2^{k+1} \mid r_{w}$ et $2^{k+1} \mid r_{w+1}$. L'intervalle $\left(r_{w+1}, r_{w}\right)$ est donc aussi une lacune de $M$, avec $r_{w+1} \geq n_{1}$ et $r_{w} \leq n$, d'une longueur $h$ telle que

$$
\begin{aligned}
h & \geq 2^{k+1}-1 \\
& \geq l \\
& \geq \max \left\{2^{d+4}, n_{j+1}-n_{j} \mid j=1, \cdots, 1+(d-1) 2^{d-2}\right\} .
\end{aligned}
$$

Ceci est contradictoire au choix de $(n, n+l]$.

\section{Fin de la démonstration du Théorème 2}

\section{Démonstration du théorème 3}

Dans cette section, on va étudier des modules instables dont la filtration nilpotente est de longueur finie. Un exemple trivial d'un tel module instable est un module instable quelconque de dimension finie. Un autre exemple est la suspension d'un module instable réduit. La cohomologie d'un groupe fini ou du classifiant d'un groupe compact vérifie aussi cette hypothèse [3].

Ci-dessous, on démontre un résultat sur la non-existence de grandes lacunes dans les modules instables connexes réalisables dont la filtration nilpotente est de longueur finie qui vérifie la condition 1.

Démonstration du Théorème 3 Comme dans la démonstration du théorème 2] il suffit de prouver l'énoncé pour les modules instables connexes dont la filtration nilpotente est de longueur finie, qui est de type $\mathcal{T}$ et vérifie la condition 1

On raisonne par l'absurde. Soit donc $M$ un module instable connexe qui est la cohomologie réduite d'un espace ou d'un spectre. Reprenons les notations 
introduites avant le théorème [3] les quotients $n_{i} M / l_{s} i_{s+1} M$ non-triviaux s'écrivent sous la forme $\Sigma^{m_{i}} R_{m_{i}}, R_{m_{i}}$ réduits, $i=1, \cdots, t, m_{1}<\cdots<m_{t}$. Tous les $R_{m_{i}}, i \in I$, soient non-triviaux dans les degrés $n_{1, i}<n_{2, i}<\cdots$. Soit $\delta$ tel que $2^{\delta} \geq t>2^{\delta-1}$. Supposons que pour $n \geq \min \left\{m_{i}+n_{1, i} \mid i \in I\right\}$, $(n, n+l]$ soit la première lacune dans $M$ de longueur

$$
l \geq \max \left\{\left(m_{t}+1\right) 2^{d+4}, n_{j+1, i}-n_{j, i} \mid i \in I, j=1, \cdots, 1+(d+\delta-1) 2^{d-2}\right\} .
$$

Soit $k$ l'unique entier $(\geq d+4)$ tel que $2^{k+1}>l \geq 2^{k}$.

Lemme 8 Pour tout $x$ tel que $|x| \leq n$, le module $\mathcal{A}_{2} x$ contient la lacune $(|x|, n+l]$.

Démonstration Pour montrer cela, on raisonne par l'absurde. A tout élément $x \in M$, on associe son degré de nilpotence, c'est-à-dire, l'entier $m_{x}$ tel que $x \in n i l_{m_{x}} M-n i l_{m_{x}+1} M$.

Soit $x$ non nul de degré maximal tel que $\mathcal{A}_{2} x$ n'est pas réduit à $\{0\}$ dans l'intervalle $(|x|, n] .\left(\mathcal{A}_{2} x\right.$ contient la lacune $(n, n+l]$ par hypothèse.)

Soit donc $y \in \mathcal{A}_{2} x$ de degré minimal tel que $y \neq 0,|x|<|y| \leq n, \bar{y} \in \Sigma^{m_{y}} R_{m_{y}}$ sa réduction que l'on note $\sigma^{m_{y}} v, v \in R_{m_{y}}$. Alors comme $\mathcal{A}_{2} y$ contient une lacune $(|y|, n+l]$ (rappelons l'hypothèse de maximalité de $x$ ), le corollaire 4 implique que $v \in \operatorname{Im}\left(S q_{0}^{k-d+2}\right)$. On sait donc que $|y|-m_{y}$ est divisible par $2^{k-d+2}$ et on écrit $|y|-m_{y}=: 2^{k-d+2} l_{y}$.

Soit de même la réduction $\bar{x} \in \Sigma^{m_{x}} R_{m_{x}}$. Notons $\bar{x}=\sigma^{m_{x}} u, u \in \operatorname{Im}\left(S q_{0}^{s}\right)-$ $\operatorname{Im}\left(S q_{0}^{s+1}\right)$.

Lemme 9 Pour $s \leq k-d, t \geq 1,2^{k-d+2}$ ne divise pas le degré de $Q_{t}^{s} u$.

Démonstration En effet on a $\left|Q_{t}^{s} u\right|=|u|+2^{s}\left(2^{t+1}-1\right)$. Considérons le $\mathcal{A}_{2}$-module engendré par $u$ dans $R_{m_{x}}$. Si $\mathcal{A}_{2} u$ contient la lacune $(|u|, n+l-$ $\left.m_{x}\right]$, le degré de $u$ est divisible par $2^{k-d+2}$, et dans ce cas $2^{k-d+2}$ ne divise pas $\left|Q_{t}^{s} u\right|$ pour $s \leq k-d$.

Supposons que $\mathcal{A}_{2} u$ ne contienne pas la lacune $\left(|u|, n+l-m_{x}\right]$ et soit $t=$ $S q^{2^{\beta}} u$ l'élément non nul du plus bas degré avec $|t| \leq n-m_{x}$. Comme $\mathcal{A}_{2} t$ contient la lacune $\left(|t|, n+l-m_{x}\right], 2^{k-d+2}|| t \mid$. Pour la même raison que dans la démonstration du lemme 5. on a $\beta \geq s$. Alors

$$
\begin{aligned}
\left|Q_{t}^{s} u\right| & =|t|-2^{\beta}+2^{s}\left(2^{t+1}-1\right) \\
& =2^{k-d+2} q-2^{\beta}+2^{s+t+1}-2^{s}
\end{aligned}
$$

et $2^{k-d+2}$ ne divise pas $\left|Q_{t}^{s} u\right|$. 
Supposons d'abord que $s \leq k-d$. Tant que $\left|Q_{t}^{s} x\right| \leq n+l$, on a donc nécessairement $Q_{t}^{s} u=0$ pour des raisons de degré. En effet si $Q_{t}^{s} u \neq 0$, $\sigma^{m_{x}} Q_{t}^{s} u=Q_{t}^{s} \bar{x}=\overline{Q_{t}^{s} x}$ la réduction de $Q_{t}^{s} x$ dans $\Sigma^{m_{x}} R_{m_{x}}$ dont le degré de nilpotence est $m_{x}$ (qui est a priori plus grand que ou égal à celui de $x$, voir [8], [10]), et dont le degré est de la forme (en appliquant le corollaire 4à la lacune $\left(\left|Q_{t}^{s} u\right|, n+l-m_{x}\right]$ dans $\left.\mathcal{A}_{2}\left(Q_{t}^{s} u\right)\right)$

$$
m_{x}+2^{k-d+2} f, \quad f \geq 0 .
$$

Donc pour que $Q_{t}^{s} u$ soit non nul, il faudrait que son degré soit multiple de $2^{k-d+2}$.

Pour tout $t$ tel que $1 \leq t \leq k-s-1$,

$$
\begin{aligned}
\left|Q_{t}^{s} x\right| & =|x|+2^{s}\left(2^{t+1}-1\right) \\
& \leq|x|+2^{s}\left(2^{k-s}-1\right) \\
& \leq n+l .
\end{aligned}
$$

Donc d'après le corollaire 3

$$
k-s-2 \leq d-2, \quad \text { soit } s \geq k-d
$$

et donc $|x|-m_{x}=: 2^{k-d} l_{x}$.

Revenons alors à l'élément $y$ non nul du plus bas degré, supérieur ou égal à $|x|+1$ dans $\mathcal{A}_{2} x$. Il est de la forme $S q^{2^{\alpha}} x$, le théorème d'Adams implique que $\alpha \leq 3$. En effet l'hypothèse de maximalité de $x$ implique que pour tout élément $z$ non nul dont le degré est entre $|x|+1$ et $n, \mathcal{A}_{2} z$ est réduit à $\{0\}$ dans l'intervalle $(|z|, n]$. Donc $S q^{2^{\alpha}} x \notin \sum_{i<\alpha} \operatorname{Im}\left(S q^{2^{i}}\right)$.

On a alors $|x|+2^{\alpha}=|y|, y \in n i l_{m_{y}} M$ et $m_{y} \geq m_{x}$. D'où, $m_{x}+2^{k-d} l_{x}+2^{\alpha}=$ $m_{y}+2^{k-d+2} l_{y}$. Donc $m_{x}+2^{\alpha}=m_{y} \bmod 2^{k-d}$. D'autre part, $2^{k+1}>l \geq$ $\left(m_{t}+1\right) 2^{d+4}$, ce qui implique que

$$
\begin{aligned}
2^{k-d}> & 8\left(m_{t}+1\right) \\
= & \left(m_{x}+m_{y}+2^{\alpha}\right)+\left(m_{t}-m_{x}\right)+\left(m_{t}-m_{y}\right)+\left(8-2^{\alpha}\right)+6 m_{t} \\
\geq & m_{x}+m_{y}+2^{\alpha} . \\
& \left|m_{x}+2^{\alpha}-m_{y}\right| \leq m_{x}+m_{y}+2^{\alpha}<2^{k-d},
\end{aligned}
$$

donc $m_{x}+2^{\alpha}=m_{y}$. Or cette égalité n'a pas de solution à cause de la condition [1. l'existence d'un tel $x$ est donc contradictoire. Donc pour tout élément $x$ de $M$ de degré inférieur à $n, \mathcal{A}_{2} x$ contient une lacune $(|x|, n+l]$.

Fin de la démonstration du Lemme 8 
On achève la démonstration en appliquant la démonstration du théorème 2 à chaque $R_{m_{i}}, i \in I$. Plus précisément, on applique la (dernière) partie de la démonstration du théorème [2] - concernant une récurrence sur la divisibilité par une puissance de 2 des degrés inférieurs à $n+l$ pour lesquels le module est non-trivial - à $R_{m_{i}}(i \in I)$. On veut donc obtenir des informations sur la divisibilité par une puissance de 2 de ses degrés inférieurs à $n+l$ auxquels on a soustrait $m_{i}$, pour lesquels $R_{m_{i}}$ est non-trivial, afin de montrer qu'on aboutit à une contradiction.

En effet, si on note $\forall i \in I$,

$$
\left(n-m_{i} \geq\right) r_{0, i}>r_{1, i}>\cdots
$$

les degrés inférieurs à $n+l-m_{i}$, pour lesquels $R_{m_{i}}$ est non-trivial, on a

$$
\forall w \geq(d+\delta-1) 2^{d-2}, \quad 2^{k+\delta+1} \mid r_{w, i} .
$$

Donc en degré inférieur ou égal à $r_{w, i}, R_{m_{i}}(i \in I)$ ne contient que des lacunes de longueur plus grande que ou égale à $2^{k+\delta+1}-1$. Donc il existe un $d_{0} \leq n$ tel qu'en degré inférieur ou égal à $d_{0}$, les $\Sigma^{m_{i}} R_{m_{i}}(i \in I)$ ne contiennent que des lacunes de longueur plus grande que ou égale à $2^{k+\delta+1}-1$, et on suppose de plus qu'il en existe au moins une en degré inférieur ou égal à $d_{0}$.

Maintenant on choisit une lacune de la plus petite longueur parmi toutes celles en degré inférieur ou égal à $d_{0}$ contenues dans l'un des $\Sigma^{m_{i}} R_{m_{i}}, i \in I$. Par le choix de cette lacune, disons $\Sigma^{m_{i_{0}}} R_{m_{i_{0}}}\left(i_{0} \in I\right)$, on sait qu'elle ne contient aucun degré en lequel $\Sigma^{m_{i_{0}}} R_{m_{i_{0}}}$ est non-trivial, et qu'elle contient au plus un degré en lequel $\Sigma^{m_{i}} R_{m_{i}}, 1 \leq i \leq t, i \neq i_{0}$, est non-trivial. Car sinon, elle contiendrait une lacune d'un des $\Sigma^{m_{i}} R_{m_{i}}, i \neq i_{0}$, en contradiction avec l'hypothèse de minimalité sur la longueur de la lacune choisie. Comme cette lacune contient au plus $t-1$ degrés en lesquels $M$ est non-trivial, il existe donc une lacune $\left(n^{\prime}, n^{\prime}+l^{\prime}\right]$, avec $n^{\prime} \geq \min \left\{m_{i}+n_{1, i} \mid i \in I\right\}$ et $n^{\prime}+l^{\prime} \leq d_{0}(\leq n)$, de longueur

$$
\begin{aligned}
l^{\prime} & \geq \frac{1}{t} \cdot 2^{k+\delta+1}-1 \\
& \geq 2^{k+1}-1 \\
& \geq l \\
& \geq \max \left\{\left(m_{t}+1\right) 2^{d+4}, n_{j+1, i}-n_{j, i} \mid i \in I,\right. \\
& \left.\quad j=1, \cdots, 1+(d+\delta-1) 2^{d-2}\right\} .
\end{aligned}
$$

Ceci est contradictoire au choix de $(n, n+l]$.

Fin de la démonstration du Théorème 3 


\section{$6 \quad$ Le cas $p$ premier impair}

On indique brièvement les résultats pour le cas $p$ premier impair. On donne d'abord les ingrédients essentiels, i.e., les opérations $Q_{t}^{s}, s, t \geq 0$, et $P_{0}^{s}, s \geq 0$. En tenant compte des signes, les formules et les résultats combinatoires sont établis de la même manière.

Définition 14 Les opérations $Q_{t}^{s}, s, t \geq 0$ sont définies récursivement comme suit:

(1) $Q_{0}^{0}=\beta$ et $Q_{t+1}^{0}=\left[P^{p^{t}}, Q_{t}^{0}\right]$, ce sont des $Q_{t}$ définis par Milnor [7];

(2) $Q_{0}^{s}=P^{p^{s-1}}$ et $Q_{t+1}^{s}=\left[P^{p^{s+t}}, Q_{t}^{s}\right], s \geq 1$.

Notation 4 Le symbole $\left(n_{1}, \cdots, n_{d}\right)=\left(2 m_{1}+\epsilon_{1}, \cdots, 2 m_{d}+\epsilon_{d}\right), \epsilon_{i}=0$ ou $1(1 \leq i \leq d)$, désigne l'élément $t^{\epsilon_{1}} u^{m_{1}} \otimes \cdots \otimes t^{\epsilon_{d}} u^{m_{d}} \in H^{*}\left(B(\mathbb{Z} / p)^{\oplus d} ; \mathbb{Z} / p\right), t$ étant de degré 1 et $u$ étant de degré 2 . Un tel élément est dit basique.

Notation 5 Comme d'habitude, $P_{0}$ désigne l'opération définie dans un module instable par

$$
P_{0} x= \begin{cases}P^{|x| / 2} x, & \text { si }|x|=0 \bmod 2 \\ \beta P^{(|x|-1) / 2} x, & \text { si }|x|=1 \bmod 2 .\end{cases}
$$

On a donc

$$
P_{0}^{s}\left(2 m_{1}+\epsilon_{1}, \cdots, 2 m_{d}+\epsilon_{d}\right)=\left(2 p^{s} m_{1}+2 p^{s-1} \epsilon_{1}, \cdots, 2 p^{s} m_{d}+2 p^{s-1} \epsilon_{d}\right),
$$

et $\operatorname{Im}\left(P_{0}^{s}\right)(s \geq 1)$ est l'ensemble des éléments $x=\sum_{j \in J}\left(2 p^{s-1} l(j)_{1}, \cdots\right.$ $\left.2 p^{s-1} l(j)_{d}\right)$ de $H^{*}\left(B(\mathbb{Z} / p)^{\oplus d} ; \mathbb{Z} / p\right)$. Par convention, $P_{0}^{0}=i d$. Ici, $J$ est un ensemble d'indices $j$ qui indexent des différents $d$-uplets $\left(l(j)_{1}, \cdots, l(j)_{d}\right)$, $l(j)_{1}, \cdots, l(j)_{d}=0,1 \bmod p, l(j)_{\alpha}$ peut être nul.

Remarque Dans toute cette section, le symbole $P_{0}^{s}$ désigne l'opération $\left(P_{0}\right)^{s}$, qui est évidemment distincte de l'opération de Milnor (utilisée dans l'appendice).

Puisqu'on a pour tout élément $x$ d'un module instable et pour tout $n \geq 1$,

$$
P^{p n} P_{0} x=P_{0} P^{n} x \quad \text { et } \quad P^{1} P_{0} x=P_{0} \beta x,
$$

on peut donc établir les propriétés de $Q_{t}^{s}, s, t \geq 0, \operatorname{sur} \operatorname{Im}\left(P_{0}^{s}\right)$ à partir de celles de $Q_{t}=Q_{t}^{0}$. Une autre façon d'établir les propriétés de $Q_{t}^{s}$ est d'utiliser le fait que

$$
\psi^{*}\left(P^{p^{s}}\right)=P^{p^{s}} \otimes 1+P^{p^{s}-1} \otimes P^{1}+\cdots+1 \otimes P^{p^{s}}
$$

devient $P^{p^{s}} \otimes 1+1 \otimes P^{p^{s}}$, une dérivation $\operatorname{sur} \operatorname{Im}\left(P_{0}^{s+1}\right)$. 
Lemme 10 Soit $x$ un élément de $H^{*}\left(B(\mathbb{Z} / p)^{\oplus d} ; \mathbb{Z} / p\right)$ tel que $x \in \operatorname{Im}\left(P_{0}^{s}\right)$ $\operatorname{Im}\left(P_{0}^{s+1}\right)$ et que $Q_{t}^{s} x=0, \forall t=q, \cdots, r$. Alors $r-q \leq d-2$.

On laisse la démonstration de ce lemme au lecteur. Voici quelques indications. D'abord, comme dans la section 3, on définit un $g$-échange $(g \geq 0)$ entre deux monômes basiques $\alpha$ et $\beta$ de la manière suivante: un tel $g$-échange existe entre $\alpha$ et $\beta$ s'il existe $i$ et $j$ tels que ces deux monômes constituent, à un ordre (entre $i$ et $j$ ) près, une paire de la forme

$$
\alpha=\left(u_{1}, \cdots, u_{i-1}, 2 u_{i}+1, u_{i+1}, \cdots, u_{j-1}, 2 u_{j}+2 p^{g}, u_{j+1}, \cdots, u_{d}\right)
$$

et $\quad \beta=\left(u_{1}, \cdots, u_{i-1}, 2 u_{i}+2 p^{g}, u_{i+1}, \cdots, u_{j-1}, 2 u_{j}+1, u_{j+1}, \cdots, u_{d}\right)$.

On dit aussi que c'est un $g$-échange en $i$-ème position pour $\alpha$ avec $\beta$. En utilisant cette nouvelle définition de $g$-échange, et les autres définitions restant inchangées, on aura la même proposition que la proposition2 pour le cas $p$ premier impair. Ensuite on achève la démonstration en construisant une $(q, r)$-classe comme dans la démonstration du corollaire 3

Lemme 11 Soit $x$ un élément de $H^{*}\left(B(\mathbb{Z} / p)^{\oplus d} ; \mathbb{Z} / p\right)$. Si $\mathcal{A}_{p} x$ contient une lacune $(|x|,|x|+l], l \geq 2(p-1) p^{k}$ pour un certain $k \geq d-3$, alors $x \in$ $\operatorname{Im}\left(P_{0}^{k-d+3}\right)$.

Démonstration Soit $\alpha$ tel que $x=P_{0}^{\alpha} x^{\prime} \in \operatorname{Im}\left(P_{0}^{\alpha}\right)-\operatorname{Im}\left(P_{0}^{\alpha+1}\right)$. Si $\alpha>k+1$, on a $\alpha \geq k-d+3$. Si $\alpha \leq k+1$, alors l'existence de la lacune dans $\mathcal{A}_{p} x$ implique que $\forall t=0, \cdots, k-\alpha$,

$$
P_{0}^{\alpha} P^{p^{t}} x^{\prime}=P^{p^{t+\alpha}} P_{0}^{\alpha} x^{\prime}=P^{p^{t+\alpha}} x=0,
$$

et que

$$
P_{0}^{\alpha} \beta x^{\prime}=P^{p^{\alpha-1}} P_{0}^{\alpha} x^{\prime}=P^{p^{\alpha-1}} x=0 .
$$

Donc $\beta x^{\prime}=0$ et $P^{p^{t}} x^{\prime}=0, \forall t=0, \cdots, k-\alpha$. D'où, $Q_{t^{\prime}} x^{\prime}=0, \forall t^{\prime}=$ $0, \cdots, k-\alpha+1$. D'après le lemme 10] on a $k-\alpha+1 \leq d-2$, d'où $\alpha \geq$ $k-d+3$.

Théorème 5 (6], [1]) Soit $X$ un espace ou un spectre, $k \geq 1$, soit $x \in$ $H^{n}(X ; \mathbb{Z} / p)$ tel que $\beta x=0$ et $P^{p^{i}} x=0, \forall i<k$, alors $P^{p^{k}} x \in \sum_{i<k} \operatorname{Im}\left(P^{p^{i}}\right)+$ $\operatorname{Im}(\beta)$. 
Définition 15 Soit $M$ un module instable infini connexe dont la filtration nilpotente est de longueur finie. Les quotients ${ }_{i l} l_{s} M / n i l_{s+1} M$ non-triviaux s'écrivent sous la forme $\Sigma^{m_{i}} R_{m_{i}}, R_{m_{i}}$ réduits, $i=1, \cdots, t, m_{1}<\cdots<m_{t}$. Notons que l'un au moins des $R_{m_{i}}$ est infini. Supposons qu'il existe des entiers $d$ et $\alpha_{d}$ tels que tous les $R_{m_{i}}$ se plongent dans $H^{*}\left(B(\mathbb{Z} / p)^{\oplus d} ; \mathbb{Z} / p\right)^{\oplus \alpha_{d}}$. Notons $I \subset\{1, \cdots, t\}$ le sous-ensemble des $i$ tels que $R_{m_{i}}$ soit infini, et soit $n_{1, i}<$ $n_{2, i}<\cdots$ les degrés en lesquels ce module est non-trivial.

Soit $\delta$ tel que $p^{\delta} \geq t>p^{\delta-1}$. Le module instable $M$ sera dit de type $\mathcal{T}$ s'il contient une lacune $(s, s+l]$ avec $s \geq \min \left\{m_{i}+n_{1, i} \mid i \in I\right\}$ et

$$
\begin{array}{r}
l \geq \max \left\{2\left(m_{t}+1\right)(p-1) p^{d+2}, n_{j+1, i}-n_{j, i} \mid i \in I,\right. \\
\left.j=1, \cdots, 1+(d+\delta)(p-1)^{2} p^{d-2}\right\} .
\end{array}
$$

Condition 2 Soit $M$ un module instable connexe dont la filtration nilpotente est de longueur finie. En utilisant les notations introduites dans la définition précédente, on dira que $M$ vérifie la condition 2$]$ si

$$
m_{j}-m_{i} \neq 1,2(p-1), \quad \forall 1 \leq i, j \leq t .
$$

Théorème 6 Soit $M$ un module qui est une suspension itérée (positive ou négative) d'un module instable connexe dont la filtration nilpotente est de longueur finie, qui est de type $\mathcal{T}$ et vérifie la condition 2. Alors $M$ n'est pas réalisable, i.e., il n'existe aucun espace ou spectre $X$ tel que $M=\tilde{H}^{*}(X ; \mathbb{Z} / p)$.

Démonstration L'idée essentielle de la démonstration de ce théorème est la même que celle de la démonstration du théorème 3. Néanmoins, certains aspects du cas d'un nombre premier impair apparaissent, non seulement on utilise le théorème 5 au lieu du théorème d'Adams, mais aussi on a besoin de reconstituer les calculs pour le cas d'un nombre premier impair. On donne dans la suite une esquisse de la démonstration de ce théorème, afin d'illustrer certains changements nécessaires par rapport à celle du théorème 3.

On note, comme dans la démonstration du théorème 3, qu'il suffit de prouver l'énoncé pour les modules instables connexes dont la filtration nilpotente est de longueur finie, qui est de type $\mathcal{T}$ et vérifie la condition 2 .

Ensuite, on raisonne par l'absurde. Supposons qu'il existe un tel module instable connexe $M$ qui est la cohomologie réduite d'un espace ou d'un spectre, et que la lacune $(n, n+l]$ soit la première dans $M$, avec $n \geq \min \left\{m_{i}+n_{1, i} \mid i \in I\right\}$, de longueur

$$
\begin{array}{r}
l \geq \max \left\{2\left(m_{t}+1\right)(p-1) p^{d+2}, n_{j+1, i}-n_{j, i} \mid i \in I,\right. \\
\left.j=1, \cdots, 1+(d+\delta)(p-1)^{2} p^{d-2}\right\},
\end{array}
$$


où $\delta$ est l'unique entier tel que $p^{\delta} \geq t>p^{\delta-1}$. Soit $k$ l'unique entier $(\geq d+2)$ tel que $2(p-1) p^{k+1}>l \geq 2(p-1) p^{k}$.

Si on note $\forall i \in I$,

$$
\left(n-m_{i} \geq\right) r_{0, i}>r_{1, i}>\cdots
$$

les degrés inférieurs à $n+l-m_{i}$, pour lesquels $R_{m_{i}}$ est non-trivial. Alors si on peut montrer que pour tout élément $x$ de $M$ de degré inférieur ou égal à $n$, $\mathcal{A}_{p} x$ contient une lacune $(|x|, n+l]$, on peut montrer par récurrence que

$$
\forall w \geq j(p-1)^{2} p^{d-2}, \quad 2 p^{k-d+j+2} \mid r_{w, i} .
$$

Puisque pour tout élément $x$ de $M$, et donc pour sa réduction $\sigma^{m_{i}} x^{\prime}$ dans $\Sigma^{m_{i}} R_{m_{i}}$, de degré inférieur ou égal à $n, \mathcal{A}_{p} x$ contient une lacune $(|x|, n+l]$ et $\mathcal{A}_{p} x^{\prime}$ contient une lacune $\left(\left|x^{\prime}\right|, n+l-m_{i}\right]$, le lemme 11 montre que le cas $j=0$ est vrai.

Supposons que c'est vrai pour $j$, alors

$$
\begin{aligned}
& \left(n+l-m_{i}\right)-r_{(j+1)(p-1)^{2} p^{d-2}, i} \\
\geq & l+r_{0, i}-r_{(j+1)(p-1)^{2} p^{d-2}, i} \\
= & l+\sum_{w=0}^{(j+1)(p-1)^{2} p^{d-2}-1}\left(r_{w, i}-r_{w+1, i}\right) \\
= & l+\sum_{h=0}^{j} \sum_{w=h(p-1)^{2} p^{d-2}}^{(h+1)(p-1)^{2}}\left(r_{w, i}-r_{w+1, i}\right) \\
\geq & 2(p-1) p^{k}+\sum_{h=0}^{j}(p-1)^{2} p^{d-2} \cdot 2 p^{k-d+h+2} \\
= & 2(p-1) p^{k}+2(p-1)^{2} \sum_{h=0}^{j} p^{k+h} \\
= & 2(p-1) p^{k}+2(p-1)\left(p^{k+j+1}-p^{k}\right) \\
= & 2(p-1) p^{k+j+1} .
\end{aligned}
$$

Puisque pour les éléments $x^{\prime}$ en degré $r_{w, i}, \mathcal{A}_{p} x^{\prime}$ contient une lacune $\left(r_{w, i}, n+\right.$ $\left.l-m_{i}\right]$, le lemme 11 montre que $2 p^{k-d+j+3} \mid r_{w, i}, \forall w \geq(j+1)(p-1)^{2} p^{d-2}$.

Rappelons que par hypothèse, il y a bien (au moins) $(d+\delta)(p-1)^{2} p^{d-2}+1$ valeurs pour l'indice $i$ de $r_{i}$. On peut donc poser $w_{0}=(d+\delta)(p-1)^{2} p^{d-2}$, alors $2 p^{k+\delta+2} \mid r_{w, i}, \forall w \geq w_{0}$. Donc en degré plus petit que $r_{w_{0}, i}, R_{m_{i}}(i \in I)$ ne contient que des lacunes de longueur plus grande que ou égale à $2 p^{k+\delta+2}-1$. Donc on peut choisir un $d_{0} \leq n$ tel qu'en degré inférieur ou égal à $d_{0}$, les $\Sigma^{m_{i}} R_{m_{i}}(i \in I)$ ne contiennent que des lacunes de longueur plus grande que ou égale à $2 p^{k+\delta+2}-1$, et on suppose de plus qu'il en existe au moins une en degré inférieur ou égal à $d_{0}$.

On peut donc choisir, comme à la fin de la démonstration du théorème 3. une lacune en degré inférieur ou égal à $d_{0}$, dans l'un des $\Sigma^{m_{i}} R_{m_{i}}(i \in I)$, telle qu'elle contient au plus $t-1$ degrés en lesquels $M$ est non-trivial. Donc il 
existe une lacune $\left(n^{\prime}, n^{\prime}+l^{\prime}\right]$, avec $n^{\prime} \geq \min \left\{m_{i}+n_{1, i} \mid i \in I\right\}$ et $n^{\prime}+l^{\prime} \leq d_{0}$ $(\leq n)$, de longueur

$$
\begin{aligned}
l^{\prime} & \geq \frac{1}{t} \cdot 2 p^{k+\delta+2}-1 \\
& \geq 2 p^{k+2}-1 \\
& >2(p-1) p^{k+1} \\
& >l \\
& \geq \max \left\{2\left(m_{t}+1\right)(p-1) p^{d+2}, n_{j+1, i}-n_{j, i} \mid i \in I,\right. \\
& \left.\quad j=1, \cdots, 1+(d+\delta)(p-1)^{2} p^{d-2}\right\} .
\end{aligned}
$$

Ceci est contradictoire au choix de $(n, n+l]$.

Pour finir la démonstration du théorème, il reste donc à montrer le

Lemme 12 Pour tout $x$ tel que $|x| \leq n$, le module $\mathcal{A}_{p} x$ contient la lacune $(|x|, n+l]$.

Démonstration Comme dans la démonstration du lemme 8, on raisonne par l'absurde. A tout élément $x \in M$, on associe son degré de nilpotence, c'est-àdire, l'entier $m_{x}$ tel que $x \in n i l_{m_{x}} M-n i l_{m_{x}+1} M$.

Soit $x$ un élément non nul de degré maximal tel que $\mathcal{A}_{p} x$ n'est pas réduit à $\{0\}$ dans l'intervalle $(|x|, n]$. Soit donc $y \in \mathcal{A}_{p} x$ de degré minimal tel que $y \neq 0$, $|x|<|y| \leq n, \bar{y} \in \Sigma^{m_{y}} R_{m_{y}}$ sa réduction que l'on note $\sigma^{m_{y}} v, v \in R_{m_{y}}$. On a, à l'aide du lemme 11] $v \in \operatorname{Im}\left(P_{0}^{k-d+3}\right)$. On sait donc que $|y|-m_{y}$ est divisible par $2 p^{k-d+2}$ et on écrit $|y|-m_{y}=: 2 p^{k-d+2} l_{y}$.

Soit de même la réduction $\bar{x} \in \Sigma^{m_{x}} R_{m_{x}}$. Notons $\bar{x}=\sigma^{m_{x}} u, u \in \operatorname{Im}\left(P_{0}^{s}\right)-$ $\operatorname{Im}\left(P_{0}^{s+1}\right)$.

Lemme 13 Pour $s \leq k-d+1, t \geq 1,2 p^{k-d+2}$ ne divise pas le degré de $Q_{t}^{s} u$.

Démonstration En effet on a

$$
\left|Q_{t}^{s} u\right|= \begin{cases}|u|+2\left(p^{t+s}-p^{s-1}\right) & s \geq 1 \\ |u|+\left(2 p^{t}-1\right) & s=0\end{cases}
$$

Considérons le module engendré par $u$ dans $R_{m_{x}}$. Si $\mathcal{A}_{p} u$ contient la lacune $\left(|u|, n+l-m_{x}\right]$, le degré de $u$ est divisible par $2 p^{k-d+2}$, et dans ce cas $2 p^{k-d+2}$ ne divise pas $\left|Q_{t}^{s} u\right|$ pour $s \leq k-d+1$.

Supposons que $\mathcal{A}_{p} u$ ne contienne pas la lacune $\left(|u|, n+l-m_{x}\right]$ et soit $t=$ $P u$ l'élément non nul du plus bas degré avec $|t| \leq n-m_{x}$. On sait que le degré de l'opération $P$ est $2(p-1) p^{\gamma}$ ou 1 . Pour la même raison que dans 
la démonstration du lemme 5, on a $\gamma \geq s$. Comme $\mathcal{A}_{p} t$ contient la lacune $\left(|t|, n+l-m_{x}\right],|t|$ est divisible par $2 p^{k-\bar{d}+2}$ et peut donc être écrit de la forme $|t|=2 p^{k-d+2} q$. Alors

$$
\left|Q_{t}^{s} u\right|= \begin{cases}2 p^{k-d+2} q-2(p-1) p^{\gamma}+2\left(p^{t+s}-p^{s-1}\right) & s \geq 1 \\ 2 p^{k-d+2} q-2(p-1) p^{\gamma}+\left(2 p^{t}-1\right) \text { ou } & \\ 2 p^{k-d+2} q-1+\left(2 p^{t}-1\right) & s=0\end{cases}
$$

et $2 p^{k-d+2}$ ne divise pas $\left|Q_{t}^{s} u\right|$.

Supposons d'abord que $s \leq k-d+1$ et que $t \geq 1$. Tant que $\left|Q_{t}^{s} x\right| \leq n+l$, on a nécessairement $Q_{t}^{s} u=0$ pour des raisons de degré. Or pour $1 \leq t \leq k-s$,

$$
\begin{aligned}
\left|Q_{t}^{s} x\right| & = \begin{cases}|x|+2\left(p^{t+s}-p^{s-1}\right) & s \geq 1 \\
|x|+\left(2 p^{t}-1\right) & s=0\end{cases} \\
& \leq \begin{cases}n+2\left(p^{k}-p^{s-1}\right) & s \geq 1 \\
n+\left(2 p^{k}-1\right) & s=0\end{cases} \\
& <n+2 p^{k} \\
& <n+2(p-1) p^{k} \\
& \leq n+l .
\end{aligned}
$$

Donc, d'après le lemme 10

$$
k-s-1 \leq d-2, \quad \text { soit } s \geq k-d+1
$$

et donc $|x|-m_{x}=: 2 p^{k-d} l_{x}$.

Maintenant on peut trouver une contradiction comme dans la démonstration du lemme 8. A l'aide du théorème 5 et de la base multiplicative de $\mathcal{A}_{p}$, on sait qu'en degré supérieur ou égal à $|x|+1$, l'élément non nul du plus bas degré dans $\mathcal{A}_{p} x$ ne peut être que $y=\beta x$ ou $y=P^{1} x$. On a alors $|x|+1=|y|$ ou $|x|+2(p-1)=|y|$. Donc

$$
m_{x}+2 p^{k-d} l_{x}+1=m_{y}+2 p^{k-d+2} l_{y}
$$

Donc, $m_{y}-m_{x}=1$ ou $2(p-1) \bmod 2 p^{k-d}$.

Puisque $2(p-1) p^{k+1}>l \geq 2\left(m_{t}+1\right)(p-1) p^{d+2}$, donc

$$
\begin{aligned}
2 p^{k-d} & >2 p\left(m_{t}+1\right) \\
& \geq 6 m_{t}+2 p \\
& >\left\{\begin{array}{l}
m_{y}+m_{x}+2(p-1) \\
m_{y}+m_{x}+1
\end{array}\right. \\
& \geq\left\{\begin{array}{l}
\left|m_{y}-m_{x}-2(p-1)\right| \\
\left|m_{y}-m_{x}-1\right|
\end{array}\right.
\end{aligned}
$$

Algebraic 83 Geometric Topology, Volume 4 (2004) 
Donc $m_{y}-m_{x}=1$ ou $2(p-1)$. Or cette égalité n'a pas de solution à cause de la condition 2 l'existence d'un tel $x$ est donc contradictoire. Donc pour tout élément $x$ de $M$ de degré inférieur à $n, \mathcal{A}_{p} x$ contient une lacune $(|x|, n+l]$.

\section{Fin de la démonstration du Lemme 12}

\section{Fin de la démonstration du Théorème 6}

\section{Appendice: Notes sur les opérations $Q_{t}^{s}$}

Dans la base de Milnor de l'algèbre de Steenrod $\mathcal{A}_{2}$, on a des opérations $Q_{t}, t \geq 0$, définies récursivement par les relations suivantes:

(1) $Q_{0}=S q^{1}$;

(2) $Q_{t+1}=\left[S q^{2^{t+1}}, Q_{t}\right]$.

Ces opérations ont des bonnes propriétés, plus précisément (Milnor, [7], §6),

Proposition 3 Soient $M, N$ deux modules instables, et soient $l, r, t \geq 0$.

(1) $\forall x \in M, Q_{r} Q_{t} x=Q_{t} Q_{r} x$ et $Q_{t}^{2} x=0$.

(2) $\forall x \in M$ et $y \in N, Q_{t}(x \otimes y)=Q_{t} x \otimes y+x \otimes Q_{t} y$.

(3) Soit u le générateur de $H^{*}(B(\mathbb{Z} / 2) ; \mathbb{Z} / 2)$ en degré 1 ,

$Q_{t} u^{2 l}=0$ et $Q_{t} u^{2 l+1}=u^{2 l+2^{t+1}}$.

Inspiré par ces propriétés, on a construit dans la section 2 les opérations $Q_{t}^{s}, s, t \geq 0$, qui possèdent aussi ces propriétés sur $\operatorname{Im}\left(S q_{0}^{s}\right)$. Dans le reste de cet appendice, on décrit quelques propriétés élémentaires de ces opérations. Puis, on donne à la fin les démonstrations du lemme 2] et du corollaire 2]

D'abord, on compare ces opérations avec les opérations connues, $P_{t+1}^{s}, s, t \geq 0$. Voici deux propriétés élémentaires:

(1) $Q_{t}^{0}=Q_{t}=P_{t+1}^{0}, Q_{0}^{s}=S q^{2^{s}}=P_{1}^{s}$.

(2) $Q_{t}^{s}$ est une opération de degré $2^{s}\left(2^{t+1}-1\right)$.

D'après ces deux propriétés, plus le fait que $P_{t+1}^{s}$ est aussi une opération de degré $2^{s}\left(2^{t+1}-1\right)$, une question curieuse est de savoir quand les deux opérations $Q_{t}^{s}$ et $P_{t+1}^{s}$ coincident. En fait, quand $s t \neq 0$, il semble que le seul cas où ces deux opérations coincident est $Q_{1}^{1}=P_{2}^{1}$.

Pour effectuer le calcul des $Q_{t}^{s}$, on note que l'on a une autre façon de définir les opérations $Q_{t}^{s}, s, t \geq 0$ :

(1) $Q_{0}^{s}=P_{1}^{s}$; 
(2) $Q_{t+1}^{s}=\left[P_{1}^{t+s+1}, Q_{t}^{s}\right]$.

Avec cette définition, on peut exprimer $Q_{t}^{s}$ en terme de la base de Milnor, à l'aide de la formule multiplicative de cette base. Voici quelques calculs qui comparent les $Q_{t}^{s}$ et $P_{t+1}^{s}$ :

(1) $Q_{1}^{1}=P_{2}^{1}, Q_{1}^{2}=P_{2}^{2}+S q(3,3), Q_{1}^{3}=P_{2}^{3}+S q(6,6)+S q(3,7)$.

(2) $Q_{2}^{1}=P_{3}^{1}+S q(7,0,1)+S q(4,1,1)$.

Pour finir cet appendice, on donne ici les démonstrations du lemme 2 et du corollaire 2

Démonstration du Lemme 2 Soient $M$ un module instable et $x$ un élément de $M$.

Quand $t=0$,

$$
Q_{0}^{s+r} S q_{0}^{s} x=S q^{2^{s+r}} S q_{0}^{s} x=S q_{0}^{s} S q^{2^{r}} x=S q_{0}^{s} Q_{0}^{r} x .
$$

Si on suppose que $Q_{t}^{s+r} S q_{0}^{s} x=S q_{0}^{s} Q_{t}^{r} x$ pour $t \leq t_{0}$, alors quand $t=t_{0}+1$, on a

$$
\begin{aligned}
Q_{t_{0}+1}^{s+r} S q_{0}^{s} x & =\left(S q^{2^{t_{0}+s+r+1}} Q_{t_{0}}^{s+r}-Q_{t_{0}}^{s+r} S q^{2^{t_{0}+s+r+1}}\right) S q_{0}^{s} x \\
& =S q^{2_{0}+s+r+1} Q_{t_{0}}^{s+r} S q_{0}^{s} x-Q_{t_{0}}^{s+r} S q^{2^{t_{0}+s+r+1}} S q_{0}^{s} x \\
& =S q^{2^{t_{0}+s+r+1}} S q_{0}^{s} Q_{t_{0}}^{r} x-Q_{t_{0}}^{s+r} S q_{0}^{s} S q^{2_{0}+r+1} x \\
& =S q_{0}^{s} S q^{2^{t_{0}+r+1}} Q_{t_{0}}^{r} x-S q_{0}^{s} Q_{t_{0}}^{r} S q^{2_{0}+r+1} x \\
& =S q_{0}^{s}\left(S q^{2^{t_{0}+r+1}} Q_{t_{0}}^{r}-Q_{t_{0}}^{r} S q^{2^{t_{0}+r+1}}\right) x \\
& =S q_{0}^{s} Q_{t_{0}+1}^{r} x .
\end{aligned}
$$

Donc, par récurrence (sur $t$ ), on a $Q_{t}^{s+r} S q_{0}^{s} x=S q_{0}^{s} Q_{t}^{r} x, \forall r, s, t$.

Démonstration du Corollaire 2 Les propriétés de $Q_{t}$ utilisées ci-dessous sont dans la proposition 3

(1) Soit $M$ un module instable. Par le lemme 2 on a $\forall x \in M$,

$$
\begin{aligned}
Q_{r}^{s} Q_{t}^{s} S q_{0}^{s} x & =Q_{r}^{s} S q_{0}^{s} Q_{t} x=S q_{0}^{s} Q_{r} Q_{t} x \\
& =S q_{0}^{s} Q_{t} Q_{r} x=Q_{t}^{s} S q_{0}^{s} Q_{r} x \\
& =Q_{t}^{s} Q_{r}^{s} S q_{0}^{s} x, \\
\text { et } \quad\left(Q_{t}^{s}\right)^{2} S q_{0}^{s} x & =Q_{t}^{s} S q_{0}^{s} Q_{t} x=S q_{0}^{s}\left(Q_{t}\right)^{2} x=0 .
\end{aligned}
$$

(2) Supposons qu'il existe $x^{\prime} \in M, y^{\prime} \in N$ tels que $x=S q_{0}^{s}\left(x^{\prime}\right), y=S q_{0}^{s}\left(y^{\prime}\right)$, alors

$$
\begin{aligned}
Q_{t}^{s}(x \otimes y) & =Q_{t}^{s}\left(S q_{0}^{s}\left(x^{\prime}\right) \otimes S q_{0}^{s}\left(y^{\prime}\right)\right) \\
& =Q_{t}^{s} S q_{0}^{s}\left(x^{\prime} \otimes y^{\prime}\right) \\
& =S q_{0}^{s} Q_{t}\left(x^{\prime} \otimes y^{\prime}\right) \\
& =S q_{0}^{s}\left(Q_{t} x^{\prime} \otimes y^{\prime}+x^{\prime} \otimes Q_{t} y^{\prime}\right) \\
& =S q_{0}^{s} Q_{t} x^{\prime} \otimes S q_{0}^{s} y^{\prime}+S q_{0}^{s} x^{\prime} \otimes S q_{0}^{s} Q_{t} y^{\prime} \\
& =Q_{t}^{s} x \otimes y+x \otimes Q_{t}^{s} y .
\end{aligned}
$$

(3) On a

$$
\begin{aligned}
Q_{t}^{s} S q_{0}^{s} u^{2 l} & =S q_{0}^{s} Q_{t} u^{2 l}=0 \\
Q_{t}^{s} S q_{0}^{s} u^{2 l+1} & =S q_{0}^{s} Q_{t} u^{2 l+1}=S q_{0}^{s} u^{2 l+2^{t+1}}=u^{2^{s}\left(2 l+2^{t+1}\right)}
\end{aligned}
$$




\section{Bibliographie}

[1] J.F. Adams, On the non-existence of elements of Hopf invariant one, Annals of Mathematics, 72 (1960), pp. 20-104.

[2] P. Gabriel, Des catégories abéliennes, Bull. Soc. Math. France 90 (1962), pp. 323-448.

[3] H.W. Henn, J. Lannes et L. Schwartz, Localizations of unstable $\mathcal{A}$-modules and equivariant mod p cohomology, Math. Ann. 301 (1995), No.1, pp. 23-68.

[4] N.J. Kuhn, On topologically realizing modules over the Steenrod algebra, Annals of Mathematics, 141 (1995), pp. 321-347.

[5] J. Lannes et L. Schwartz, Sur la structure des $\mathcal{A}$-modules instables injectifs, Topology (1989), Vol.28, No.2, pp. 153-169.

[6] A. Liulevicius, The factorization of cyclic reduced powers by secondary cohomology operations, Mem. A.M.S. 42 (1962).

[7] J. Milnor, The Steenrod algebra and its dual, Annals of Mathematics, 67 (1958), pp. 150-171.

[8] L. Schwartz, La filtration nilpotente de la categorie $\mathcal{U}$ et la cohomologie des espaces de lacets, Algebraic topology - rational homotopy (Louvain-la-Neuve, 1986), pp. 208-218, Lecture Notes in Math., 1318, Springer, Berlin (1988).

[9] L. Schwartz, Unstable modules over the Steenrod algebra and Sullivan's fixed point set conjecture, Chicago Lectures in Mathematics Series (1994).

[10] L. Schwartz, La filtration de Krull de la catégorie $\mathcal{U}$ et la cohomologie des espaces, Algebr. Geom. Topol. 1 (2001), pp. 519-548 (electronic).

[11] N. Shimada et T. Yamanoshita, On triviality of the $\bmod p$ Hopf invariant, Japan J.Math. 31 (1961), pp. 1-25.

LAGA, Institut Galilée, Université Paris Nord

93430 Villetaneuse, France

Email: donghua.jiang@polytechnique.org

Received: 23 September 2002 Revised: 5 September 2003 